\title{
CONFINEMENT ET DÉCONFINEMENT ÉNERGÉTIQUE DES SECTEURS DU BÂTIMENT ET DES TRANSPORTS
}

\author{
Auteur : \\ Richard CANTIN \\ Ecole Nationale des Travaux Publics de l'Etat - Université de Lyon \\ Rue Maurice Audin 69120 Vaulx-en-Velin - France \\ richard.cantin@entpe.fr
}

\section{Résumé :}

En imposant le confinement des populations, la pandémie du Covid-19 a brutalement freiné de nombreuses activités économiques. Rapidement, les impacts sociaux, environnementaux et climatiques se sont fait sentir sur tous les continents.

La prise de conscience des conséquences de cette pandémie et du confinement interroge les façons de consommer, de travailler, de se déplacer et d'habiter, les modes de vie présents et futurs. Ainsi, dans l'incertitude, se posent une énième fois les questions relatives aux stratégies de croissance ou de décroissance pour le XXI siècle et notamment celles relatives à la mise en æuvre d'une transition énergétique.

En effet, dans de nombreux pays, le secteur du bâtiment, très consommateur d'énergie, peine toujours à engager effectivement sa transition énergétique et avance à petits pas. Quant au secteur des transports, important émetteur de gaz à effet de serre, durement éprouvé par le confinement, il semble finalement contraint de s'y engager mais à marche forcée. Dans ce contexte, penser le monde après la pandémie invite à s'interroger sur les difficultés qu'ont ces deux secteurs économiques majeurs à faire leur transition énergétique.

L'article a pour objectif de mettre en évidence des réflexions prospectives permettant de repenser les problématiques énergétiques des secteurs du bâtiment et des transports. Pour cela, l'approche systémique est mise en ouvre afin d'appréhender la complexité du confinement des consciences dans des modèles à penser les questions énergétiques. Des exemples montrent les limites de ces modèles. Ils illustrent le confinement dans le modèle thermo-industriel, dans des biais cognitifs et la prégnance des macro-systèmes techniques du passé. Enfin, des pistes d'un déconfinement énergétique sont proposées afin d'évaluer les conditions épistémologiques d'une transition énergétique complexe.

\section{Mots-clés :}

Energie, climat, industrie, bâtiment, transport, prospective

\section{INTRODUCTION}

En imposant le confinement des populations, la pandémie du Covid-19 a brutalement freiné de nombreuses activités. La prise de conscience des impacts économiques, sociaux et environnementaux s'est rapidement fait sentir. Elle interroge les façons de consommer, de travailler, de se déplacer et d'habiter, les modes de vie présents et futurs.

Alors que les enjeux liés à la production, la transformation, le stockage, le transport et l'utilisation des énergies sont complexes, la crise a modifié en profondeur la consommation énergétique mondiale. Dans de nombreux pays, les secteurs les plus consommateurs d'énergie que sont ceux du bâtiment et des transports ont été fortement impactés (Chevalier, 2004 ; Quénard, 2011 ; Gerlache, 2019 ; MTE, 2020 ; IEA, 2020).

Avant cette crise, le secteur des transports était responsable d'un quart des émissions directes de $\mathrm{CO}_{2}$ dues principalement à la combustion d'énergies fossiles. Avec une augmentation des émissions de gaz à effet de serre des secteurs des transports maritime et aérien, le trafic routier représentait les trois quarts des émissions du secteur des transports (IEA, 2020). 
Pendant la crise, le transport longue distance a connu une réduction spectaculaire de son activité, avec une baisse de $60 \%$ du trafic aérien en 2020 et de $30 \%$ de la demande ferroviaire. Dans les villes, les habitants délaissent les transports publics, qui sont en baisse de $50 \%$ dans certains pays, pour se tourner vers les voitures privées et les mobilités douces comme la marche et le vélo (IEA, 2020).

Avant la crise, le secteur du bâtiment consommait plus du tiers de la consommation finale d'énergie mondiale. Il était responsable d'environ $40 \%$ des émissions de $\mathrm{CO}_{2}$. Depuis longtemps, l'industrie de la construction est un important consommateur de ressources et d'énergies non renouvelables (Spence \& Mulligan, 1995). La demande d'énergie liée à la construction et à l'exploitation des bâtiments a continué d'augmenter dans le monde à cause des nouvelles constructions, d'un meilleur accès à l'énergie dans les pays en développement et d'une plus grande utilisation d'appareils consommateurs d'énergie (IEA, 2020).

Pendant la crise, le secteur du bâtiment connaît un report partiel de la demande d'énergie du tertiaire vers le résidentiel. Le télétravail et la distanciation sociale réduisent l'utilisation des bâtiments tertiaires et augmentent les activités consommatrices d'énergie à domicile (IEA, 2020 ; Cantin et al., 1998). Au premier semestre 2020, la consommation d'électricité dans les bâtiments résidentiels de certains pays a augmenté de $20 \%$ à $30 \%$ tout en diminuant d'environ $10 \%$ dans les bâtiments tertiaires. La baisse des prix des énergies fossiles a prolongé de 10 à $40 \%$ le retour sur investissement des principales mesures d'efficacité énergétique réduisant leur attractivité par rapport à d'autres investissements (IEA, 2020).

Ces deux secteurs sont presque totalement dépendants de ressources disponibles en quantités limitées (Club de Rome, 1992 IEA, 2020 ; GIEC, 2020). Dans tous les pays, les secteurs du bâtiment et des transports sont systémiquement dépendants l'un de l'autre, même si les enjeux et les problématiques de transition énergétique de chaque secteur sont différents (Aykut et Evrard, 2017 ; Collard, 2018 ; IEA, 2020 ; MTE, 2020). Traiter les enjeux des transitions énergétiques de ces secteurs séparément les uns des autres, sans vision globale, ne peut mener qu'à des résultats peu satisfaisants, et vu la diversité des enjeux, il est pertinent de recourir à une approche intégrative (Gerlache, 2019).

Compte tenu de la complexité révélée par la crise du Covid-19 quant à l'avenir des secteurs du bâtiment et les transports, cette article a pour objectif de mettre en évidence, par une approche systémique, quelques difficultés qu'ont ces deux secteurs pour s'engager dans leurs transitions énergétiques. Les caractéristiques du confinement dans un macro-système thermo-industriel déterminé par une rationalisation numérique sont présentées. Quelques pistes de réflexion pour un déconfinement sont ensuite esquissées afin de repenser les problématiques énergétiques de ces deux secteurs.

\section{CONFINEMENT DANS UN MACRO-SYSTÈME THERMO-INDUSTRIEL}

Ces deux secteurs forment des macro-systèmes techniques qui constituent des infrastructures de la vie quotidienne (Gras, 1997). Ils réunissent des ensembles complexes d'éléments reliés par des réseaux d'échanges de flux de natures diverses, de matières, de personnes, de produits, d'énergies, d'informations, etc. Ils combinent des objets industriels, une organisation de la distribution des flux, et des entreprises de gestion commerciale reliant l'offre et la demande (Gras, 1997). Ils initient et façonnent les modes de bâtir, d'habiter, de se déplacer et de transporter.

Depuis deux siècles, ils sont structurés, fonctionnent et évoluent en consommant principalement les énergies fossiles produites par la combustion du charbon, du pétrole et du gaz naturel. La dépendance à ces énergies non renouvelables est presque totale pour les secteurs du bâtiment et des transports. Cette dépendance est à appréhender en considérant l'histoire des techniques, les choix industriels et les politiques publiques du passé (Gille, 1978; Daumas, 1996; Chevalier, 2004 ; Gras, 2007 ; Belot, 2015). Depuis le XVIII ${ }^{\mathrm{e}}$ siècle, ces macro-systèmes organisent une économie fondée sur un modèle thermo-industriel visant à dominer la nature par la recherche de la vitesse et de la puissance (Gille, 1978 ; Gras, 2007).

Le choix du feu produit par les énergies fossiles comme moyen de puissance a été une rupture dans l'histoire des techniques. En privilégiant la combustion, le modèle thermo-industriel a limité l'exploration d'autres voies technologiques non thermiques (Gras, 2007). Pendant deux siècles, 
l'exploitation de ressources énergétiques renouvelables issues de la terre, du soleil, de l'eau et de l'air ont été délaissées.

Avant le recours massif aux énergies fossiles, les sources d'énergie étaient associées à l'exploitation des êtres vivants. Les humains et les animaux tiraient eux même leur énergie des végétaux (Gras, 2007). Pour les transports terrestres, la puissance animale a longtemps constitué l'unique ressource alors que la puissance du vent a été, pour le transport maritime, la principale ressource énergétique de la navigation à voiles. Pour les bâtiments, les ressources disponibles localement ont été, pendant des siècles, ingénieusement exploitées par l'architecture bioclimatique et vernaculaire, comme en témoigne l'important patrimoine bâti ancien existant dans le monde. Par ailleurs, les moulins à eau et les barrages ont assuré une part importante de la force motrice des machines (Gras, 2007).

Avec les énergies fossiles, les activités industrielles ont prospéré en imposant leurs modèles énergétiques. Sans fortes contraintes énergétiques et environnementales, les secteurs du bâtiment et des transports se sont développés avec le modèle industriel. La vitesse et la puissance étaient plus facilement accessibles avec ces énergies (Gras, 2007 ; Illich, 2008).

Aujourd'hui, le macro-système thermo-industriel est intrinsèquement lié aux énergies fossiles et aux systèmes financiers existants (Giraud, 2020). Il a induit différents imaginaires et modèles à penser (Illich, 2008; Musso, 2014; Musso, 2017). S'est développée la logique de produit où l'industriel conçoit et fabrique des produits en fonction de ce qu'il sait et de ce qu'il anticipe du marché. Il vend ce qu'il a produit (importance de la fonction commerciale et marketing). L'organisation des tâches facilitent le recours aux machines (industrie de l'automobile et industrie du bâtiment). Il produit en usine, selon un processus intégré et maîtrisé en totalité, avec une main d'œuvre spécialisée mais non qualifié au sens de la maîtrise d'un métier (Chemillier, 2002).

Cette logique industrielle du produit principalement développée dans le secteur de l'automobile est différente de la logique de projet développée dans le secteur du bâtiment (Chemillier, 2002 ; Cantin \& Cryonnet, 2014). L'architecte conçoit un projet en fonction du programme et du terrain de son client. Le programme est spécifique à chaque terrain et client. La production s'effectue au coup par coup. Elle suit la commande, sans certitude de continuité. Les entreprises sont consultées pour réaliser le projet sur le terrain. Plusieurs entreprises (lots) sans lien permanent avec l'architecte sont sollicitées. Le chantier est l'unité de production, toujours nouvelle et éphémère, soumise aux aléas climatiques (Chemillier, 2002 ; Cantin \& Cryonnet, 2014).

L'industrie combine une légitimité, une normativité managériale et une puissante institution, l'entreprise, qui peut rivaliser avec l'Etat (Musso, 2017 ; Rappin, 2019). L'entreprise-usine devient la technico-institution économique de l'industrie dont la normativité est fixée par le management au nom de l'efficacité. La mesure et le calcul favorisent une quantification du monde par la recherche de la performance (Musso, 2017).

Le macro-système thermo-industriel est ainsi le résultat d'un processus d'industriation fait de rationalisation et d'abstraction, l'imaginaire industriel remaniant en tout sens la dialectique HommeMachine (Chaplin, 1936 ; Musso, 2014, 2020).

La crise du Covid-19 a mis en évidence l'addiction aux énergies fossiles du macro-système thermoindustriel qui organise les secteurs du bâtiment et des transports. Ce macro-système s'accompagne d'un management cybernétique (mi-robot mi-humain) qui induit la numérisation, le chiffrage et finalement accélère le développement des systèmes numériques. 


\section{DÉTERMINISME D'UNE RATIONALISATION NUMÉRIQUE}

Avec la pandémie du Covid-19, les systèmes numériques d'information et de communication connaissent un essor sans précédent. Ils impactent les secteurs du bâtiment et des transports, les façons d'habiter et de se déplacer. Ils modifient les rapports humains. La Machine s'introduit avec le télétravail, la multiplication des interfaces et des artefacts de communication et le confinement numérique. Les technologies de l'information et de la communication déterminent une rationalisation qui permet de mesurer, automatiser et planifier les relations, les échanges et les représentations.

Dans les secteurs du bâtiment et des transports, se développent les infrastructures numériques, l'internet des objets, les capteurs, les compteurs, les caméras, les radars, les actionneurs, les véhicules connectés, les drones, les machines et matériels informatiques, les logiciels, etc. Ces systèmes numériques mobilisent de multiples parties prenantes (BTP, électronique, électricité, télécommunications, etc.). Ils forment un réseau de communication porteur de logiques algorithmiques et cybernétiques (Benasayag, 2019). Ils produisent des mesures, des données, des indicateurs et des informations qui déterminent les représentations et les modèles à penser.

Parmi les mesures utilisées pour évaluer l'impact de la crise, le Produit Intérieur Brut (PIB) est un exemple d'une rationalisation numérique. Il quantifie une valeur économique et comptabilise sans distinction les activités constructives ou positives mais aussi destructives ou négatives (accidents industriels, routiers, etc.). Il est utilisé pour rationaliser et simplifier la perception de situations complexes : "Grâce aux campagnes de vaccination, à l'adoption de politiques sanitaires concertées et aux aides financières publiques, le PIB mondial devrait augmenter de $4.2 \%$ en 2021 après avoir reculé de $4.2 \%$ cette année. Si les vaccins sont déployés plus rapidement, dopant la confiance et atténuant l'incertitude, la reprise sera plus vigoureuse. À l'inverse, des retards dans la vaccination, des difficultés à contenir de nouvelles poussées épidémiques et une incapacité à tirer les leçons de la première vague assombriraient les perspectives" (OCDE, 2020).

Or, les limites du PIB sont connues depuis longtemps (Besançon, 2013). Par exemple, il ne prend pas en compte les différentes conditions de production, la qualité des services rendus, des activités non marchandes ou non administratives telles que les activités artistiques ou les activités bénévoles. Alors que ces activités essentielles ont été fortement impactées par la crise du Covid-19, il convient de s'interroger sur la dépendance des modèles à penser vis-à-vis de systèmes d'information et de communication en pleine essor.

De même, l'indicateur simple de la consommation énergétique d'un bâtiment ou d'un véhicule n'est pas une mesure représentative de sa performance globale puisqu'il n'indique pas le niveau de confort pour l'habitant ou le conducteur. Ainsi, toute mesure des émissions de gaz à effet de serre (par exemple en tonne d'équivalent $\mathrm{CO}_{2}$ ), du bilan carbone (en kilogramme équivalent carbone), de l'empreinte écologique (en hectare global) ou de la consommation énergétique (en $\mathrm{kWhep} / \mathrm{m}^{2}$.an ou en litre $/ \mathrm{km}$ ) ne peut avoir de sens que si elle est contextualisée et explicitement renseignée sur les conditions de son élaboration et de son utilisation.

Depuis longtemps, cette rationalité est quantifiée par la mesure du temps (Musso, 2017). Cette mesure du temps, de la vitesse et de la performance, se définit avec et pour le modèle thermo-industriel. Celuici cherche la domination et la transformation de la nature, en s'appuyant sur une rationalité managériale silencieuse, accompagnée de chiffres. Celle-ci permet la rencontre de la cybernétique et $\mathrm{du}$ management, et les concepts de la cybernétique sont appliqués à la production industrielle. Estimant pouvoir réduire les incertitudes et améliorer la gestion des bâtiments et des transports, l'homme transfère une partie de son pouvoir décisionnel à ces modèles numériques façonnés par les machines et par les logiciels (Benasayag, 2019).

Avant cette numérisation accélérée par la crise du Covid-19, une entreprise de rationalisation appelée le New Public Management a déterminé des principes d'organisation analytique et non systémique (Bezes \& Musselin, 2015). Dans les années 1990, cette entreprise a donné lieu à un mouvement d'agencification, puis à un mouvement de fusion dans les années 2000. L'un vise à confier les missions étatiques à des structures autonomes. Celles-ci ont des responsabilités renforcées et soumises à une gestion par les résultats. L'autre soutient des objectifs managériaux tels que la réduction des coûts et 
des effectifs ou la diffusion d'instruments de mesure de la performance. Qu'il s'agisse d'agencification ou de fusion, ces réorganisations des structures étatiques approfondissent une composante de la bureaucratie, la spécialisation. Ainsi une rationalité instrumentale prévaut dans les méthodes managériales reposant sur des croyances dans l'efficacité de l'organisation (Bezes \& Musselin, 2015).

Avec la rationalisation numérique, ce modèle managérial est porté pour transformer les relations entre les entités administratives, notamment en les contractualisant, pour en mesurer leur effets, leur efficacité et leur performance. Cette rationalisation favorise la diffusion d'un cadre managérial et de principes d'organisation analytique. Au sein des administrations publiques, les conventions remplacent les relations hiérarchiques. Les modalités d'attribution des moyens sont indexées aux résultats (nombre d'actes réalisés par exemple) plutôt qu'aux données contextuelles (nombre d'agents réalisant des actes), ou s'effectuent de manière sélective par mise en concurrence entre les services. Ces processus ont souvent été associés à des formes de privatisation ou de marchandisation de la sphère publique. Ce qui apparaît alors est au moins autant la marchandisation du secteur public que la poursuite, sous une forme renouvelée, de sa rationalisation (Audria, 2004 ; Bezes, 2005 ; Barone et al., 2018 ; Pesqueux, 2020).

Cette rationalisation numérique et managériale favorise l'évolution des systèmes d'information et de communication dans les secteurs du bâtiment (Gestion technique des bâtiments, Building Information Modeling, bâtiments intelligents, etc.) et des transports (Systèmes de transport intelligents, véhicules connectés, véhicules autonomes, etc.).

Avec la crise du Covid-19, l'asservissement numérique, les restrictions de liberté, d'échanger et de circuler, éprouvent ces méthodes de management et mettent en tension le macro-système thermoindustriel. Apparaissent les processus à l'œuvre qui contraignent les représentations, les formes de management et les modèles à penser.

\section{COMPLEXIFIER POUR DECONFINER}

Le déconfinement suppose de retrouver les libertés de se reconnecter avec l'environnement pour habiter et se déplacer. La prise de conscience des changements globaux, accélérée avec cette crise, invite à penser différemment (Claeys, Lambert \& Piecq, 2017). Les nouveaux défis invitent à penser au-delà des frontières du macro-système existant.

Pour cela, l'approche systémique fournit les concepts et une nouvelle grille de lecture pour explorer les pistes d'un déconfinement (Rosnay, 1975; Durand, 1979; Le Moigne, 1994; Morin, 1997 ; Donnadieu et al., 2003). Elle interroge les modèles à penser et aide à appréhender les biais cognitifs de représentations parfois réductrices. Elle appelle une problématisation qui nécessite une modélisation responsabilisant le modélisateur (Le Moigne, 2020b). Cette problématisation demande une réévaluation des solutions du passé, des connaissances et des modèles existants. Elle exige une critique analytique et systémique des modèles à penser.

Les activités des secteurs du bâtiment et des transports générées par le modèle thermo-industriel du passé témoignent d'un modèle économique et intellectuel complexe (Musso 2017). Leur logique industrielle est portée par un secteur économique ouvert. Pour fonctionner et durer, elle considère que les entrées et les sorties du macro-système thermo-industriel, que sont les ressources et les déchets, sont des "stocks illimités" (figure 1).

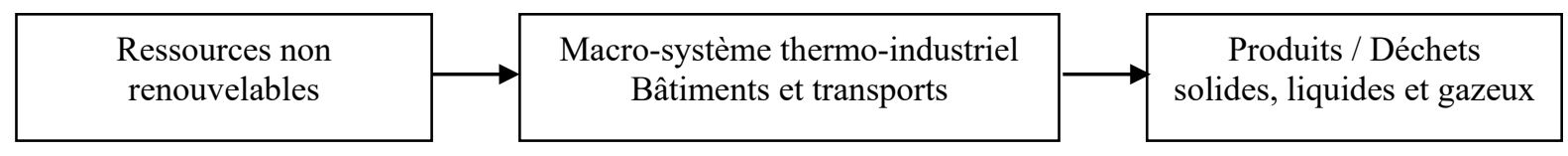

Figure 1. Modèle linéaire de causalités directes sans environnement 
Ce modèle à penser permet d'imaginer une croissance illimitée. Les activités des secteurs du bâtiment et des transports ont longtemps été conduites selon cet enchaînement linéaire de causalités directes. Depuis un demi siècle, ce modèle qui ne prend pas en compte les interactions avec un environnement est remis en question (Club de Rome, 1972).

Pour conduire un déconfinement énergétique des secteurs du bâtiment et des transports, il est possible d'environner le modèle économique confiné dans le macro-système thermo-industriel. Une piste consiste à rétablir les liens entre les activités économiques et l'environnement (Figure 2).

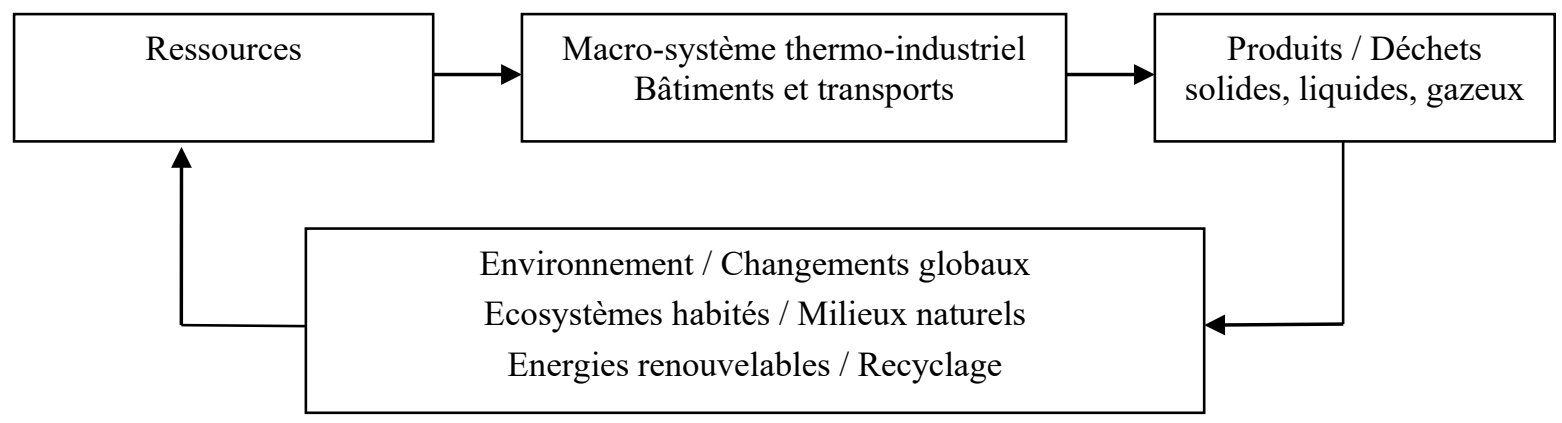

Figure 2. Modèle d'un système économique environné

Ce modèle d'un système économique environné met en évidence par une boucle de rétroaction l'existence de liens entre les sorties (effets) et les entrées (causes) du macro-système. Les activités des secteurs du bâtiment et des transports épuisent les ressources et génèrent le changement climatique et diverses pollutions qui modifient fortement l'environnement. Ces changements impactent rétroactivement les activités de ces secteurs, les façons d'habiter et de se déplacer. Cette boucle traduit l'effet boomerang ou l'effet rebond, les effets étant liés aux causes.

Ce modèle révèle les principes d'une économie circulaire, globale et solidaire, régulée et complexe qui intègre naturellement l'environnement. Ces principes systémiques dominaient l'économie bien avant le $\mathrm{XVIII}^{\mathrm{e}}$ siècle. Un déconfinement énergétique des secteurs du bâtiment et des transports selon de tels principes signifie donc une complexification du modèle existant.

Par ailleurs, le modèle linéaire de causalités directes ne permet pas de représenter l'émergence d'évènements incertains ou imprévus. Il suppose que les connaissances et les actions sont parfaitement maîtrisées.

Le modèle illustré avec la figure 1 est un exemple de ce type de modèle comme celui de la figure 3 . Ce dernier modélise la financiarisation simple et prévisible des connaissances et des actions issues d'un diagnostic.

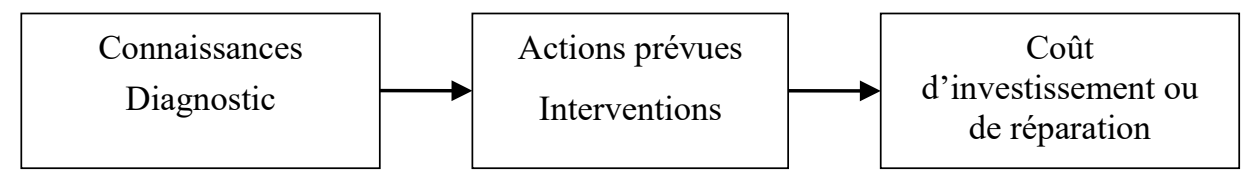

Figure 3. Modèle linéaire séquentiel simple et prévisible 
Le diagnostic produit des connaissances qui conduisent à définir des actions. Celles-ci sont ensuite traduites en coût d'investissement ou de réparation. Ce modèle linéaire est cependant source d'erreurs. Il n'intègre pas l'incomplétude de connaissances et l'environnement d'où proviennent les perturbations incertaines ou imprévues. La crise de Covid-19 a révélé ce biais cognitif aussi appelé l'effet iceberg. L'effet iceberg interroge tout diagnostic d'une situation complexe car le diagnostic dépend des limites du champ de connaissances de l'observateur. Ces limites peuvent être dépassées par la complexification du modèle linéaire en ajoutant une boucle de rétroaction qui révèle d'autres coûts induits (Figure 4).

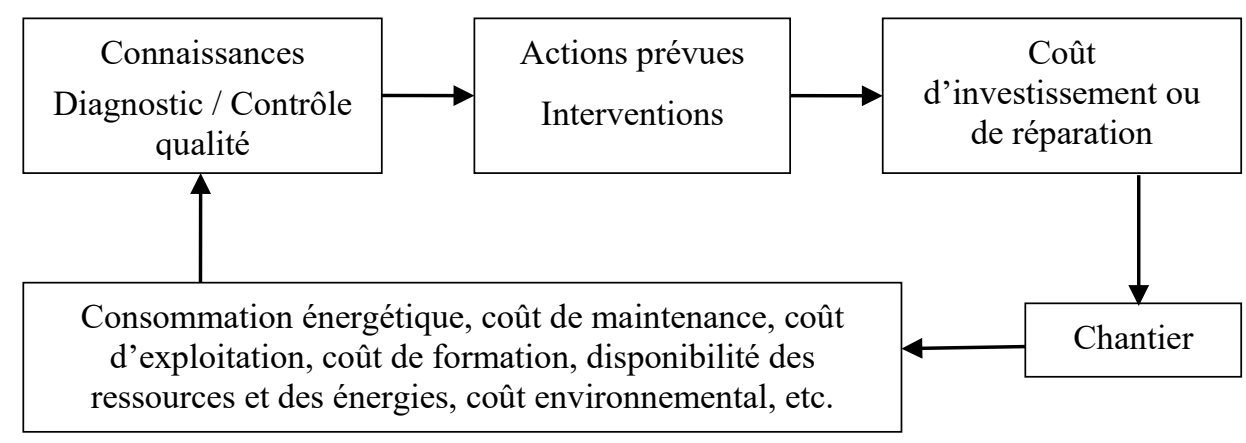

Figure 4. Déconfinement par une boucle de rétroaction du modèle linéaire simple

Compléter le modèle avec une boucle de rétroaction permet d'interroger l'environnement d'où peuvent provenir les perturbations et de réguler les interventions.

Ces exemples montrent que des pistes de déconfinement des modèles à penser consistent à complexifier les modèles linéaires, en y intégrant l'environnement des systèmes.

Il peut s'agir d'introduire l'environnement dans le modèle économique pour en faire un modèle global, circulaire et régulé (Figures 1 et 2). Dans ce cas, le macro-système thermo-industriel existant et les choix énergétiques des secteurs du bâtiment et des transports doivent être repensés pour éviter les effets boomerang ou les effets rebond.

Il peut aussi s'agir d'augmenter le champ de connaissances des diagnostics pour réduire les effets iceberg (Figures 3 et 4). Dans ce cas, les modèles de production et de diffusion des connaissances doivent être repensés afin de réorienter et renforcer les activités de recherche et de formation pour accompagner le déconfinement énergétique des deux secteurs majeurs de l'économie.

\section{CONCLUSION}

La crise du Covid-19 révèle la très faible capacité de résilience énergétique des secteurs du bâtiment et des transports confinés dans un modèle thermo-industriel du passé dépendant principalement des énergies fossiles.

En délaissant les énergies renouvelables pendant deux siècles, le macro-système thermo-industriel a formaté un modèle économique basé sur les énergies fossiles. Il a structuré les secteurs du bâtiment et des transports, les confinant dans un modèle énergétique qui s'épuise.

Les systèmes d'information et de communication bénéficiant de la crise sont porteurs d'une rationalisation numérique. Celle-ci contraint les organisations et bouleverse les systèmes humains et sociaux. La modification des réseaux, des logiques d'organisation et de management, des flux d'information, d'énergie et de matière menace l'équilibre de nombreux systèmes économiques, sociaux et environnementaux (Atlan, 1979).

Dans le passé, d'autres périodes de crises et d'incertitudes ont été marquées par la recherche de nouvelles réponses organisationnelles, techniques et énergétiques (Chevallier, 2004; Bouvier \& 
Laborie, 2016). Ces expériences rappellent que la complexité des crises rend la réflexion prospective nécessaire.

Compte tenu de la multiplicité et de la variété des facteurs d'évolution des deux secteurs principaux consommateurs d'énergies fossiles, il est nécessaire de développer les études prospectives (Jouvenel, 1999 ; Godet, 1991 ; Gaudin, 1990). Si le modèle énergétique est resté globalement invariant au XX siècle, le champ des possibles peut être exploré en anticipant les effets systémiques induits par les choix énergétiques.

Pour les acteurs des secteurs du bâtiment et des transports, l'évolution des systèmes énergétiques peut être étudiée sur le long terme en anticipant les effets des changements environnementaux. La crise climatique marque la fin logique de la société thermo-industrielle et le recours aux énergies renouvelables exige la soumission au moins partielle à un ordre naturel situé hors d'une politique industrielle (Gras, 2007).

Les choix énergétiques intégrant les effets des changements climatiques ne peuvent être dictées par une urgence résultant d'actions inefficaces ou de l'inaction (Jouvenel, 1999). Le déconfinement énergétique peut être conduit avec des approches interdisciplinaires s'appuyant sur la connaissance des techniques et de leurs impacts depuis deux millénaires et non deux siècles (Gille, 1978 ; Daumas, 1996).

Le confinement dans des solutions du passé et dans des approches réductrices de la problématique énergétique, décontextualisées des changements globaux en cours, doit être questionné. L'absence de résultats probants depuis plusieurs années doit interroger non seulement la qualité effective des solutions du passé mais aussi les difficultés à apporter de nouvelles réponses. Par exemple, le très faible nombre de bâtiments autonomes en énergie et l'augmentation de la précarité énergétique interrogent l'efficacité des politiques publiques pré-Covid-19.

Pour accompagner un déconfinement énergétique, les problématiques interdisciplinaires de rationalisation numérique des organisations sont à repenser (Atlan, 1979; Bachelard, 1995; Morin, 1995, 1997, 2005). Ainsi, les choix d'une transition numérique dans les secteurs du bâtiment et des transports doivent être éprouvés par l'évaluation de leurs effets systémiques.

Les activités de recherche visant à produire les nouvelles connaissances indispensables à une transition énergétique pour les bâtiments et les transports n'ont pas été une priorité pour un secteur de l'énergie s'inscrivant dans des enjeux dépassant la coopération des Etats. Les stratégies d'approvisionnement, les prix pratiqués, les techniques mises en oeuvre avec les intérêts industriels afférents, ont contribué à faire de l'énergie un secteur dans lequel s'expriment les intérêts nationaux et une collaboration qui se révèle parfois utopique (Bouvier \& Laborie, 2016 ; Rappin, 2019).

Pour ces raisons, le déconfinement énergétique passe également par l'investissement massif dans une recherche publique et une formation pluridisciplinaire repensée (Gaudin, 1978; Morin, 2000 ; Miramond, 2003 ; Poisson \& Clenet, 2005 ; Le Moigne, 2020a). Si de nombreux acteurs peuvent y contribuer, les gouvernements ont un rôle essentiel à jouer. Les initiatives émanant de la société, des jeunes et moins jeunes, des entreprises et des investisseurs sont déterminantes. Toutefois les gouvernements disposent de la plus grande capacité à façonner un destin énergétique. Ce sont eux qui fixent les conditions et les investissements dans le secteur de l'énergie, dans les secteurs du bâtiment et des transports, secteurs qui peuvent porter un déconfinement énergétique. C'est vers les gouvernements que le monde se tourne pour obtenir des signaux clairs et une orientation sans équivoque quant à la trajectoire à suivre (IEA, 2020).

\section{REFERENCES}

ATLAN, H. (1979). Entre le cristal et la fumée. Le Seuil. Paris.

AUDRIA, R. (2004). New Public Management et Transparence : essai de déconstruction d'un mythe actuel. Thèse, Université de Genève, Suisse.

AYKUT, S. C. et EVRARD, A. (2017). Une transition pour que rien ne change ? Changement institutionnel et dépendance au sentier dans les "transitions énergétiques" en Allemagne et en France. De Boeck Supérieur. Revue internationale de politique comparée. 
BACHELARD, G. (1995). Le nouvel esprit scientifique. Ed. Quadrige. Paris.

BARONE, S. MAYAUX, P-L. et GUERRIN, J. (2018). Introduction. Que fait le new public management aux politiques environnementales? ARPoS | "Pôle Sud" 2018/1 nº 48.

BELOT, R. (2015). L'Atome et la France. Aux origines de la technoscience francaise. Odile Jacob.

BENASAYAG, M. (2019). La tyrannie des algorithmes. Editions textuel.

BESANCON, Y. (2013). L'hégémonie anachronique du PIB. Réseau Canopé. Idées économiques et sociales. 2013/3 $\mathrm{N}^{\circ} 173$.

BEZES, P. \& MUSSELIN, C. (2015). Le new public management. Entre rationalisation et marchandisation? Une French touch dans l'analyse des politiques publiques ? Éditeur : Presses de Sciences Po.

BEZES, P. (2005). Le renouveau du contrôle des bureaucraties: L'impact du New Public Management. Informations sociales 2005/6 ( $\left.\mathrm{n}^{\circ} 126\right)$.

BOUVIER, Y. \& LABORIE, L. (2016). L'Europe en transitions. Energie, mobilité, communication XVIIIe - XXIe siècles. Editions Nouveau Monde.

CANTIN, R. MICHEL, P et GUARRACINO, G. (1998). Impact of teleworking on indoor climate at home. Lyon, EPIC'98.

CANTIN, R. \& CRYONNET, J.C. (2014). De l'interprétation systémique du projet de réhabiliter un bâtiment. Union Européenne de Systémique. Acta European Systemica.

CANTIN, R. \& CRYONNET, J.C. (2017). Stratégies d'identification des data utiles à la conduite d'opérations de construction. Union Européenne de Systémique. Acta European Systemica.

CHAPLIN, C. (1936). Les temps modernes. Modern times. Film.

CHEMILLIER, P. (2012). L'épopée de l'industrialisation du bâtiment après la guerre 1939-1945. Texte de la conference du 14 juin 2002. Comité d'histoire. Ministère de l'Equipement, des Transports, de l'Aménagement du territoire, du Tourisme et de la Mer.

CHEVALIER, J-M. (2004). Les grandes batailles de l'énergie. Gallimard.

CLAEYS, D., LAMBERT, C. \& PIECQ, A. (eds.). (2017). Thinking outside of the box! How does systemic thinking help creative, inventive and change processes? Acta Europeana Systemica, $\mathrm{n}^{\circ} 07$.

CLUB DE ROME.(1972). Halte à la croissance? Rapport sur les limites de la croissance. Paris: Fayard.

COLLARD, F. (2018). La politique énergétique en Europe. CRISP Centre de recherche et d'information socio-politiques. Courrier hebdomadaire du CRISP. 2018/38.

DAUMAS, M. (1996). Histoire générale des techniques. Quadrige / Presses UNiversitaires de France.

DONNADIEU, G. DURAND, D. NEEL, D. NUNEZ, E. et SAINT-PAUL, L. (2003) L'approche systémique : de quoi s'agit-il ? Diffusion de la pensée complexe. Travaux du groupe AFSCET.

DONNADIEU, G. \& KARSKY, M. (2004). La systémique. Penser et agir dans la complexité. Editions Liaisons.

DURAND, D. (1979). La systémique. PUF.

GAUDIN, T. (1978). L'écoute des silences. Les institutions contre l'innovation. Paris : union générale d'édition.

GAUDIN, T. (dir.) (1990). 2100 récit du prochain siècle. Paris: Payot.

GENG, Y. JI, W. WANG, Z. LIN, B. and ZHU, Y.(2019). A review of operating performance in green buildings: Energy use, indoor environmental quality and occupant satisfaction. Energy \& Buildings.

GERLACHE, J. de. (2019). Mettre en oeuvre les transistions énergétiques. Stratégie intégrative et gestion opérationnelle. Dunod.

GIEC (2020). Groupe d'experts Intergouvernemental sur l'Evolution du Climat. https://www.ipcc.ch/

GILLE, B. (1978). Histoire des Techniques. Paris: Encyclopédie de La Pléiade. 
GIRAUD, G. (2020). Quel modèle économique pour la transition écologique? La conversation scientifique par Etienne Klein. [en ligne sept. 2020] https://www.franceculture.fr/emissions/laconversation-scientifique/quel-modele-economique-pour-la-transition-ecologique

GODET, M. (1991). De l'anticipation à l'action. Paris: Dunod.

GRAS, A. (1997). Les macro-systèmes techniques. Presses Universitaires de France.

GRAS, A. (2007). Le choix du feu. Aux origines de la crise climatique. Fayard.

IEA, (2020). International Energy Agency. Reports. (2020). https://www.iea.org/

ILLICH, I. (2018). Energie et équité. Flammarion, Paris.

JOUVENEL, H. de. (1999). La démarche prospective - un bref guide méthodologique. Futuribles.

LE MOIGNE J.L. (1994). La théorie du système général. PUF. Paris.

LE MOIGNE, J.L. (2020a). Problématiser devient le maître mot et enseigner à problématiser devient une mission essentielle de l'enseignement. Réseau Intelligence de la Complexité - MCX-APC.

LE MOIGNE, J. L. (2020b). Problématiser, c'est d'abord modéliser en responsabilisant le modélisateur. Réseau Intelligence de la Complexité - MCX-APC.

MIRAMOND, M. (2003). Pour un atelier permanent Complexité-Transdisciplinarité au sein d'une école d'ingénieur. Grand atelier MCX. La formation au défi de la complexité. Lille.

MOLES, A.(1995). Les sciences de l'imprécis. Paris: Seuil, 1995. 360 p.

MORIN, E. (1997). La méthode. La nature de la nature. Le Seuil. 1997.

MORIN, E. (2000). Les sept savoirs nécessaires à l'éducation du futur. Ed. Seuil. Paris. 2000. 130p.

MORIN, E. (2005). Introduction à la pensée complexe. Editions du Seuil, Paris, 2005.

MTE, (2020). Ministère de la Transition Ecologique. https://www.ecologie.gouv.fr/

MUSSO, P. (2014). L'imaginaire industriel. Editions Manucius.

MUSSO, P. (2017). La religion industrielle. Monastère, manufacture, usine. Une généalogie de l'entreprise. Fayard.

OCDE. (2020). Les perspectives s'éclaircissent, mais la reprise sera graduelle. Perspectives économiques de l'OCDE, décembre 2020. https://www.oecd.org/perspectives-economiques

PESQUEUX, Y. (2020). New Public Management (NPM) et Nouvelle Gestion Publique (NGP). Doctorat. France. 2020

POISSON, D. \& CLENET, J. (2005). Complexité de la formation et formation à la complexité. Ingenieum L'Harmattan.

QUENARD, D. (2011). Se loger, se déplacer : peut-on se libérer de l'addiction aux énergies fossiles? La chimie et l'habitat. EDP Sciences.

RAPPIN, B. (2019). De l'industrialisme Saint-Simonien aux fondateurs du management scientifique: l'utopie de la coopération. L'Harmattan. Revue Française d'Histoire des Idées Politiques.

ROSNAY Joël de. (1975). Le macroscope - Vers une vision globale. Le Seuil. Paris

SPENCE, R. \& MULLIGAN, H. (1995). Sustainable development and the construction industry. Habitat Intl. Vol. 19. No 3. 